\title{
Graphene Nanoribbons in Electrochemical Sensors and Biosensors: A Review
}

Umamaheswari Rajaji ${ }^{1}$, Rameshkumar Arumugam ${ }^{2}$, Shen-Ming Chen ${ }^{1, *}$, Tse-Wei Chen ${ }^{1}$, Tien-Wen Tseng', Sathishkumar Chinnapaiyan ${ }^{3}$, Shih-Yi Lee, ${ }^{4, *}$ Wen-Han Chang, ${ }^{5,6,7}$

${ }^{1}$ Department of Chemical Engineering and Biotechnology, National Taipei University of Technology, No.1, Section 3, Chung-Hsiao East Road, Taipei 106, Taiwan

${ }^{2}$ Department of Chemistry, Bannari Amman Institute of Technology, Sathyamangalam, Erode, India

${ }^{3}$ Department of Mechanical \& Electrical Engineering, National Taipei University of Technology,

No.1, Section 3, Chung-Hsiao East Road, Taipei 106, Taiwan.

${ }^{4}$ Division of Pulmonary and Critical Care Medicine, MacKay Memorial Hospital; MacKay Medicine, Nursing and Management College.

${ }^{5}$ MacKay Memorial College Department of Cardiology, MacKay Memorial Hospital, Taiwan.

${ }^{6}$ Department of Emergency Medicine, MacKay Memorial Hospital; Institute of Mechatronic Engineering, National Taipei University of Technology, Taiwan

${ }^{7}$ Graduate Institute of Injury Prevention and Control, Taipei Medical University; Department of Medicine, Taiwan

*E-mail: smchen1957@gmail.com (S.-M.Chen), Leesyi5538@ yahoo.com.tw (S.-Y. Lee)

doi: $10.20964 / 2018.07 .51$

Received: 13 March 2018 / Accepted: 10 May 2018 / Published: 5 June 2018

Recently, graphene nanoribbons (GNRs) are narrow strips of graphene sheets with width in nanometers $(<50 \mathrm{~nm})$ and they are becoming attractive material in a variety of electrochemical applications owing to their outstanding electronic and catalytic properties. The most fascinating property of GNRs that distinguish them from other members of graphene family is their higher areanormalized edge-plane structures. In addition, GNRs have large surface area, high conductivity, residual oxygen functionalities, and accessible sites for catalysis, biocompatibility and good stability. Therefore, GNRs based nanomaterials are recently getting much popularity in electrochemically sensing and biosensing applications. In this review, we are presenting a summary of recent reports on GNRs based nanomaterials for electrochemical sensors and biosensors.

Keywords: Graphene, Layered nanomaterials, Electroanalytical chemistry, Electrochemical sensors and biosensors, Modified electrodes

\section{FULL TEXT}


(C) 2018 The Authors. Published by ESG (www.electrochemsci.org). This article is an open access article distributed under the terms and conditions of the Creative Commons Attribution license (http://creativecommons.org/licenses/by/4.0/). 